\title{
Phase Transition in Hot Pion Matter
}

\author{
A. Kostyuk ${ }^{1,2}$, M. Gorenstein ${ }^{1,2}$, H. Stöcker ${ }^{1}$ and W. Greiner ${ }^{1}$ \\ ${ }^{1}$ Institut für Theoretische Physik, Universität Frankfurt, Germany \\ ${ }^{2}$ Bogolyubov Institute for Theoretical Physics, Kyiv, Ukraine
}

\begin{abstract}
The equation of state for the pion gas is analyzed within the third virial approximation. The second virial coefficient is found from the $\pi \pi$-scattering data, while the third one is considered as a free parameter. The proposed model leads to a first-order phase transition from the pion gas to a more dense phase at the temperature $T_{p t}<136 \mathrm{MeV}$. Due to relatively low temperature this phase transition cannot be related to the deconfinement. This suggests that a new phase of hadron matter — 'hot pion liquid' — may exist.
\end{abstract}

The widely used thermal models of the hadron gas (HG) are mostly based on the ideal gas approach. To take into account attractive interactions between hadrons, the hadron resonances are included in the ideal gas picture [1]. This method, describing quite satisfactory the gaseous phase, does not lead to a phase transition (PT). In phenomenological models, PT is usually introduced 'by hand': it is postulated from the very beginning that two different phases, for instance HG and quark gluon plasma, exist; each phase has its own equation of state and Gibbs criterion is used to find the PT point.

It is interesting to study whether a PT can naturally follow from the equation of state of real HG without any ad hoc assumptions. This would allow us to describe, at least at a qualitative level, both phases within a unique approach and to estimate the PT parameters using available hadron data.

In the present letter we make the first step in this direction: we study the equation of state of the pion matter, i.e. we ignore (anti-)nucleons and strange particles.

Our starting point is the equation of state for a gas of interacting particles in the form of virial expansion [2 [4]:

$$
p(T, n)=T \sum_{i=1}^{\infty} a_{i}(T) n^{i} .
$$

It allows to calculate the gas pressure $p$ at given temperature $T$ and particle density $n$, provided that the virial coefficients $a_{i}(T)$ are known.

In the case of a relativistic system, like a hot pion gas, the particle number is not conserved, hence it is inconvenient to choose $n$ as an independent variable. On the other hand, the chemical potential $\mu$ for pions in isotopically symmetric systems at chemical equilibrium is equal to zero. Therefore, it is more convenient to choose $T$ and $\mu$ as independent variables: $n=n(T, \mu=0)$ should be found from Eq.(II) in thermodynamically consistent way.

Let us express the thermodynamical quantity of the gas satisfying Eq.(17) via $T$ and $\mu$. The particle density is given by the formula 


$$
n(T, \mu)=\frac{\partial p(T, \mu)}{\partial \mu}
$$

Using the equation of state (1) leads to a differential equation for $n(T, \mu)$ :

$$
n(T, \mu)=\frac{\partial p(T, n)}{\partial n} \frac{\partial n(T, \mu)}{\partial \mu}=T \sum_{i=1}^{\infty} i a_{i}(T) n^{i-1}(T, \mu) \frac{\partial n(T, \mu)}{\partial \mu} .
$$

Integration of this equation yields

$$
\mu=T\left[a_{1}(T) \log [C(T) n(T, \mu)]+\sum_{i=2}^{\infty} \frac{i}{i-1} a_{i}(T) n^{i-1}(T, \mu)\right],
$$

where $C(T)$ is the integration constant. In the low density limit $n \rightarrow 0$ at fixed temperature (which is equivalent to $\mu \rightarrow-\infty$ ) the equation (4) should reproduce the properties of the ideal classical gas, i.e. $n(T, \mu)$ should coincide with the absolute activity $z(T, \mu)$, which is given by the formula [3]:

$$
z(T, \mu) \equiv g \phi(T ; m) \exp \left(\frac{\mu}{T}\right)
$$

where $g$ is the (iso-)spin degeneracy factor ( $g=3$ for the pion), $m$ is the pion mass, and $\phi(T ; m)$ can be expressed via the $K_{2}$ modified Bessel function

$$
\phi(T ; m)=\frac{1}{2 \pi^{2}} \int_{0}^{\infty} p^{2} d p \exp \left(-\frac{\sqrt{p^{2}+m^{2}}}{T}\right)=\frac{m^{2} T}{2 \pi^{2}} K_{2}\left(\frac{m}{T}\right) .
$$

This allows to fix the integration constant. Taking into account that $a_{1}(T) \equiv 1$ one gets

$$
C(T)=\frac{\exp \left(\frac{\mu}{T}\right)}{z(T, \mu)} \equiv \frac{1}{g \phi(T ; m)},
$$

Finally, one has the following transcendental equation for $n(T, \mu)$

$$
z(T, \mu)=n(T, \mu) \exp \left[\sum_{i=1}^{\infty} \frac{i+1}{i} a_{i+1}(T) n^{i}(T, \mu)\right] .
$$

To apply this equation to the pion gas it is necessary to know the values of the virial coefficients. The second virial coefficient is straightly related to the second Mayer's cluster integral [2, 4]: $a_{2}=-b_{2}$, which is given by

$$
b_{2}=b_{2}^{(0)}+b_{2}^{(i)}
$$

where the first term

$$
b_{2}^{(0)}=\frac{1}{2 g} \frac{\pi^{2}}{2 g m^{2} T} \frac{K_{2}(2 m / T)}{\left[K_{2}(m / T)\right]^{2}}
$$

appears due to Bose effects. It exists in an ideal Bose gas too. The second term 


$$
b_{2}^{(i)}=\frac{2 \pi}{m^{4} T^{2}\left[g K_{2}(m / T)\right]^{2}} \sum_{I=0}^{2} \sum_{L}^{\prime}(2 I+1)(2 L+1) \int_{0}^{\infty} d \varepsilon \varepsilon^{2} \delta_{L, I}(\varepsilon) K_{1}\left(\frac{\varepsilon}{T}\right) .
$$

is related to the interactions between particles. The function $\delta_{L, I}(\varepsilon)$ stands for the phase shift [5] 8 ] of $\pi \pi$-scattering in the state with angular momentum $L$, isospin $I$ and center-ofmass energy $\varepsilon$. The sum over $L$ runs over those values, which ensure symmetry of the total two pion wave function, i.e.

$$
L=\left\{\begin{array}{l}
0,2,4,6, \ldots \text { for } I=0,2 \\
1,3,5,7, \ldots \text { for } I=1
\end{array}\right.
$$

The second cluster integral $b_{2}$ was calculated in [9]. The dependence of $a_{2}$ on the temperature is shown in Fig.11 (see Ref. [9] for details).

A model independent determination of all other virial coefficients $a_{i}(T), i>2$ is hardly possible. The quantum theory is developed only for $a_{3}(T)$ [10], but lacking of detailed experimental information on the three-pion interaction makes it impossible to use this theory for the case of pion gas. For the higher virial coefficients, the problem looks even more hopeless.

A simple model will be adopted for the further consideration:

- The virial series is truncated after the third term: $a_{i}(T) \equiv 0, i>3$;

- The third virial coefficient $a_{3}(T)$, at fixed $T$, is considered as a free parameter.

It is convenient to introduce the notation $\xi(T) \equiv a_{3}(T) /\left[a_{2}(T)\right]^{2}$ and treat the dimensionless quantity $\xi(T)$ as a free parameter. In this case, the equation of state (8) takes the form

$$
p(T, n(T, \mu))=T n(T, \mu)\left\{1+a_{2}(T) n(T, \mu)+\xi(T)\left[a_{2}(T) n(T, \mu)\right]^{2}\right\},
$$

where the value of $n(T, \mu)$ is found from the transcendental equation

$$
z(T, \mu)=n(T, \mu) \exp \left[2 a_{2}(T) n(T, \mu)+\frac{3}{2} \xi(T)\left[a_{2}(T) n(T, \mu)\right]^{2}\right] .
$$

Let us analyze the behavior of the r.h.s. of Eq.(14) at some temperature $T$, as a function of $n>0$. For convenience we denote it by $f(n)$. Three distinct cases are possible (see Figs. 2 and 3 ):

1. $\xi(T) \leq 0$. The function $f(n)$ has a single extremum - a maximum at the point

$$
n_{a}= \begin{cases}\frac{1}{2\left|a_{2}\right|} & \text { if } \xi(T)=0 \\ \frac{\sqrt{1+3|\xi(T)|}-1}{3|\xi(T)|\left|a_{2}\right|} & \text { if } \xi(T)<0,\end{cases}
$$

2. $\xi(T) \geq 1 / 3$. The function $f(n)$ grows monotonically for all $n>0$. 
3. $0<\xi(T)<1 / 3$. In this case the function $f(n)$ has two extrema: a maximum at the point

$$
n_{a}=\frac{1-\sqrt{1-3 \xi(T)}}{3 \xi(T)\left|a_{2}\right|}
$$

and a minimum at the point

$$
n_{b}=\frac{1+\sqrt{1-3 \xi(T)}}{3 \xi(T)\left|a_{2}\right|} .
$$

In the first case, the values of $f(n)$ are bound from the above: $f(n) \leq f\left(n_{a}\right)$. As $z(T, \mu)$ is a monotonically increasing function of the temperature, the equation (14) has no solution at high temperatures $T>T_{a}$, where the temperature $T_{a}$ is found from the equation

$$
z\left(T_{a}, \mu\right)=f\left(n_{a}\right) .
$$

The numerical calculations give $T_{a} \leq 128 \mathrm{MeV}$ assuming chemical equilibrium $(\mu=0)$. (The equality is reached at $\xi\left(T_{a}\right)=0$.) This means that if the third virial coefficient of the pion gas is negative at the temperatures $T \gtrsim 128 \mathrm{MeV}$, the model cannot describe the pion matter at these temperatures. Consideration of higher order terms of virial expansion would be necessary to obtain a satisfactory equation of state of the pion gas in this case.

At $T<T_{a}$ the equation (14) has two solutions: $n_{g}$ and $n_{x}, n_{g}<n_{a}<n_{x}$ (See Fig. (2). It is easy to check that the derivative of the pressure with respect to the density is negative at the point $n_{x}$ :

$$
\left.\frac{\partial p(T, n)}{\partial n}\right|_{n=n_{x}}<0 .
$$

This means that the root $n_{x}$ corresponds to an unstable phase, which cannot exist, because any small fluctuations of the density would destroy it. The solution $n_{g}$ corresponds to a stable phase which can be obviously interpreted as a pion gas.

The interpretation of the second case is straightforward. The equation (14) has a unique solution at any temperature, which describes the gaseous phase of the pion matter. No PT takes place.

The third case is the most interesting. The equation (14) has solutions at any temperature. At low temperatures, $T<T_{b}$, where $T_{b}$ satisfies the condition $z\left(T_{b}, \mu\right)=f\left(n_{b}\right)$, the equation (14) has a single solution $n_{g}\left(n_{g}<n_{a}\right)$, which can be obviously interpreted as the density of gaseous phase. As it is shown in Fig. 3, at intermediate temperatures $T_{b}<T<T_{a}, T_{a}$ is given by Eq.(18), two more solutions appear: $n_{x}\left(n_{a}<n_{x}<n_{b}\right)$ and $n_{l}$ $\left(n_{l}>n_{b}\right)$. Similar to the second case, the root $n_{x}$ corresponds to an unstable phase, which cannot exist. In contrast, the points $n_{g}$ and $n_{l}$ give a positive derivative of the pressure and correspond to (meta-)stable phases. The questions which of two phases is stable or metastable is resolved by the Gibbs criterion: the pressure of a stable phase is higher than that of metastable one. The point, when the two pressures are equal to each other 


$$
p\left(T_{p t}, n_{g}\left(T_{p t}, \mu\right)\right)=p\left(T_{p t}, n_{l}\left(T_{p t}, \mu\right)\right)
$$

is a PT point. It is reminiscent of the first order gas-liquid PT. At high temperatures $T>T_{a}$ the equation (14) has an unique solution corresponding to this 'liquid' phase.

In spite of its similarity to the gas-liquid PT, which is well known from molecular physics , there is an essential difference. In molecular physics, the stable liquid exists at temperatures below the PT point $\left(T<T_{p t}\right)$, while high temperatures $\left(T>T_{p t}\right)$ correspond to the gaseous phase. Here we observe the opposite situation: at low temperatures $\left(T<T_{p t}\right)$ the pion matter only slightly differs from the ideal classical pion gas. The dense 'liquid' phase appears at high $\left(T>T_{p t}\right)$ temperatures and thus can be called 'hot pion liquid'.

There are two reasons that explain the qualitative difference in the behavior of the pion matter as compared with ordinary substances known from molecular physics:

- In contrast to the number of molecules, the number of pions is not conserved and rapidly decreases as temperature falls down. So that the density of isotopically symmetrical pion matter becomes so small at low temperatures that the interactions between the particles plays almost no role, which corresponds to a perfect gas.

- In molecular physics the dependence of the second virial coefficient on the temperature typically can be approximated by the formula [4] $a_{2}(T)=\beta-\frac{\alpha}{T}$, where nonnegative constants $\alpha$ and $\beta$ come, respectively, from attractive and repulsive parts of the intermolecular potential. It is easily seen that at high temperatures the repulsive part becomes dominating and the virial coefficient becomes positive. In contrast, the second virial coefficient of the pion gas remains negative at high temperatures (see Fig.(1)), which indicates the attractive nature of $\pi \pi$-interaction.

As was mentioned above, we study the pion matter at chemical equilibrium. Thus the dense phase of pion matter is distinct from multipion droplets considered in Ref. [11]. They may exist only at $\mu>0$ at low temperature and evaporate if the temperature exceeds some critical value, similarly to ordinary liquid.

The dependence of the particle density on the temperature is shown in Fig. ઔ. The value of the free parameter $\xi(T) \equiv 0.21$ was arbitrary chosen from the interval $0<\xi(T)<1 / 3$ to provide an example. As is seen from the figure, the density of the gaseous phase is only slightly different from the ideal gas density indicating that virial expansion can be safely applied in this region. On the other hand, the density of the 'liquid' phase is about one order of magnitude higher than that of the pion gas, so one should not expect that the present model can provide quantitative description of the 'pion liquid'. It rather illustrates the features of PT at a qualitative level.

At the same time, to estimate the upper bound of the PT temperature it is sufficient to analyze the properties of the gaseous phase. In fact, $T_{p t}$ cannot exceed the maximum temperature of the overheated gas $T_{p t}<T_{a}$ (see Fig. 3). The upper bound for $T_{a}$ is approached at $\xi\left(T_{a}\right) \rightarrow 1 / 3$ and comprises $136 \mathrm{MeV}$. At $\xi(T) \geq 1 / 3$ the phase transition, as was mentioned above, disappears. That is, our model states that if a first order PT does exist in the isotopically symmetric equilibrium pion matter, it takes place at the temperature not higher than $136 \mathrm{MeV}$.

A similar first order PT in hadron matter but at higher temperature $(T=190 \div 200 \mathrm{MeV})$ was predicted long ago within various models [12,13]. In Ref. [12] the hot and dense phase 
was interpreted as quark-gluon plasma, i.e. the PT was identified with color deconfinement PT. This agrees with the estimations of the temperature of the deconfinement PT from lattice simulations of two-flavor QCD: $T_{d e c}=150-200 \mathrm{MeV}$ 14,15.

In contrast, the present model predicts a PT at lower temperature, which cannot be identified with the deconfinement PT. This suggest the possibility that a new phase of hadron matter, distinct from the HG and QGP may exist at zero baryonic chemical potential in the temperature range $136 \mathrm{MeV} \lesssim T \lesssim T_{d e c}$. It is formed from colorless pions attracting to one another rather than from deconfined quarks and gluons and can be called 'hot pion liquid'.

It is possible that the 'hot pion liquid' can be created in relativistic heavy ion collisions at RHIC and LHC energies, where the pions will play a dominant role at the final stage of the reaction.

The authors are grateful to Larry McLerran for fruitful discussion and his interest to the paper and to Gephard Zeeb for reading the manuscript and usefull comments. The financial support of GSI and DAAD (Germany) is appreciated. The research described in this publication was made possible in part by Award No. UP1-2119 of the U.S. Civilian Research \& Development Foundation for the Independent States of the Former Soviet Union (CRDF). 


\section{REFERENCES}

[1] R. Dashen, S.K. Ma, H.J. Bernstein Phys. Rev. 187, 345 (1969).

[2] K. Huang, "Statistical mechanics" John Wiley 65 Sons (1963).

[3] J.E. Mayer and M. Goeppert Mayer, "Statistical mechanics" John Wiley 65 Sons (1977).

[4] W. Greiner, L. Neise and H. Stöcker "Thermodynamik und Statistische Mechanik" Verlag Harri Deutsch (1993).

[5] B. Hyams et al., Nucl. Phys. B64, 134 (1973).

[6] S. Ishida, M. Ishida, H. Takahashi, T. Ishida, K. Takamatsu and T. Tsuru, particle," Prog. Theor. Phys. 95, 745 (1996) hep-ph/9610325.

[7] S. Ishida, T. Ishida, M. Ishida, K. Takamatsu and T. Tsuru, Prog. Theor. Phys. 98, 1005 (1997).

[8] S. Ishida, hep-ph/9905260.

[9] A. Kostyuk, M. Gorenstein, H. Stocker and W. Greiner, hep-ph/0004163.

[10] A. Pais and G.E. Uhlenbeck, Phys. Rev. 116, 250 (1959).

[11] I. N. Mishustin and W. Greiner, J. Phys. G G19 (1993) L101.

[12] R. Hagedorn and J. Rafelski, CERN-TH-2947 Invited lecture given at Int. Symp. on Statistical Mechanics of Quarks and Hadrons, Bielefeld, Germany, Aug 24-31, 1980.

[13] J. Theis, G. Graebner, G. Buchwald, J. A. Maruhn, W. Greiner, H. Stöcker and J. Polonyi, Phys. Rev. D28 (1983) 2286.

[14] T. Blum, L. Karkkainen, D. Toussaint and S. Gottlieb, Phys. Rev. D51 (1995) 5153 hep-lat/9410014.

[15] W. Greiner and D. H. Rischke, Phys. Rept. 264 (1996) 183. 


\section{FIGURES}

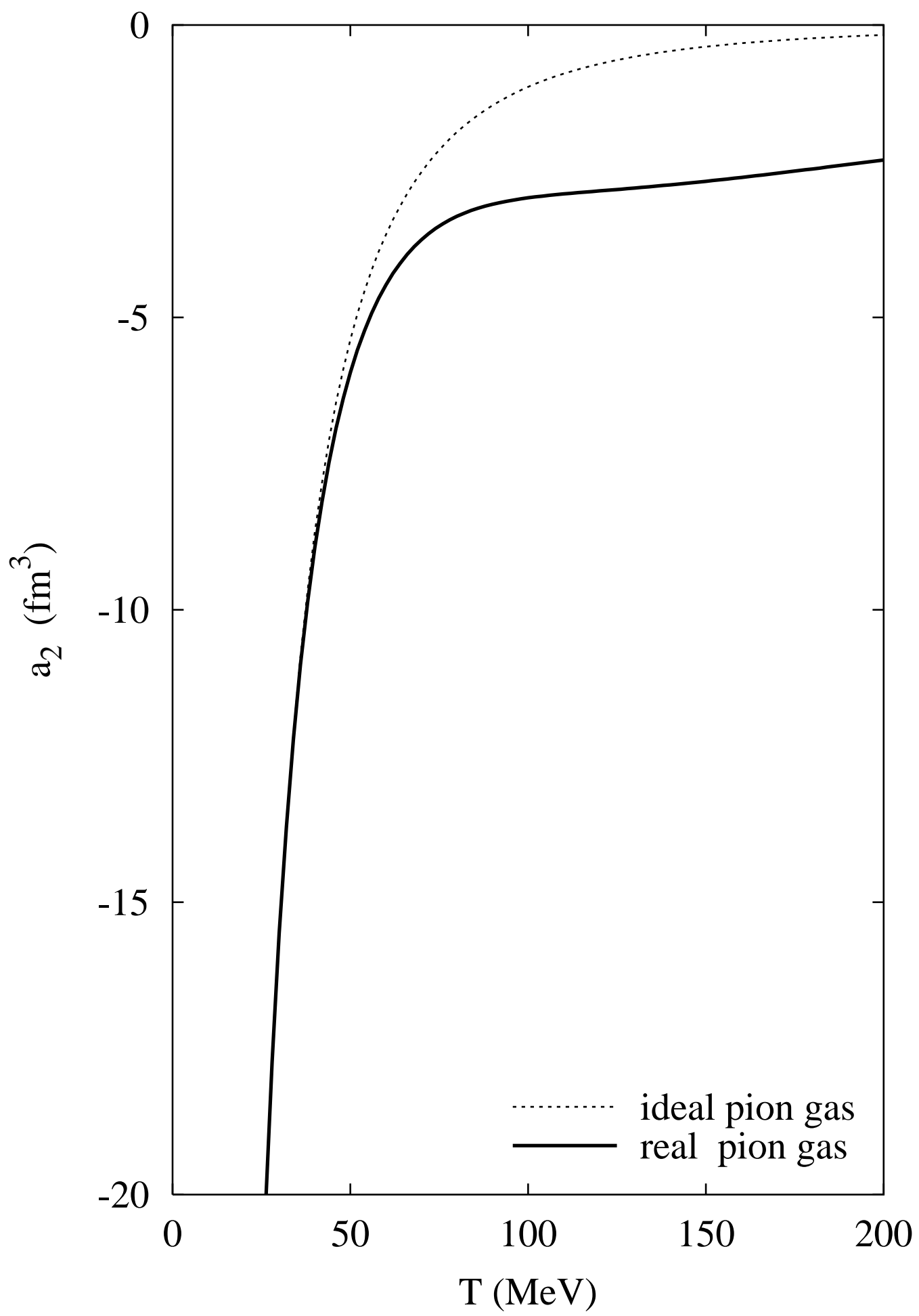

FIG. 1. The dependence of the second virial coefficient of the ideal and real pion gas on the temperature. 


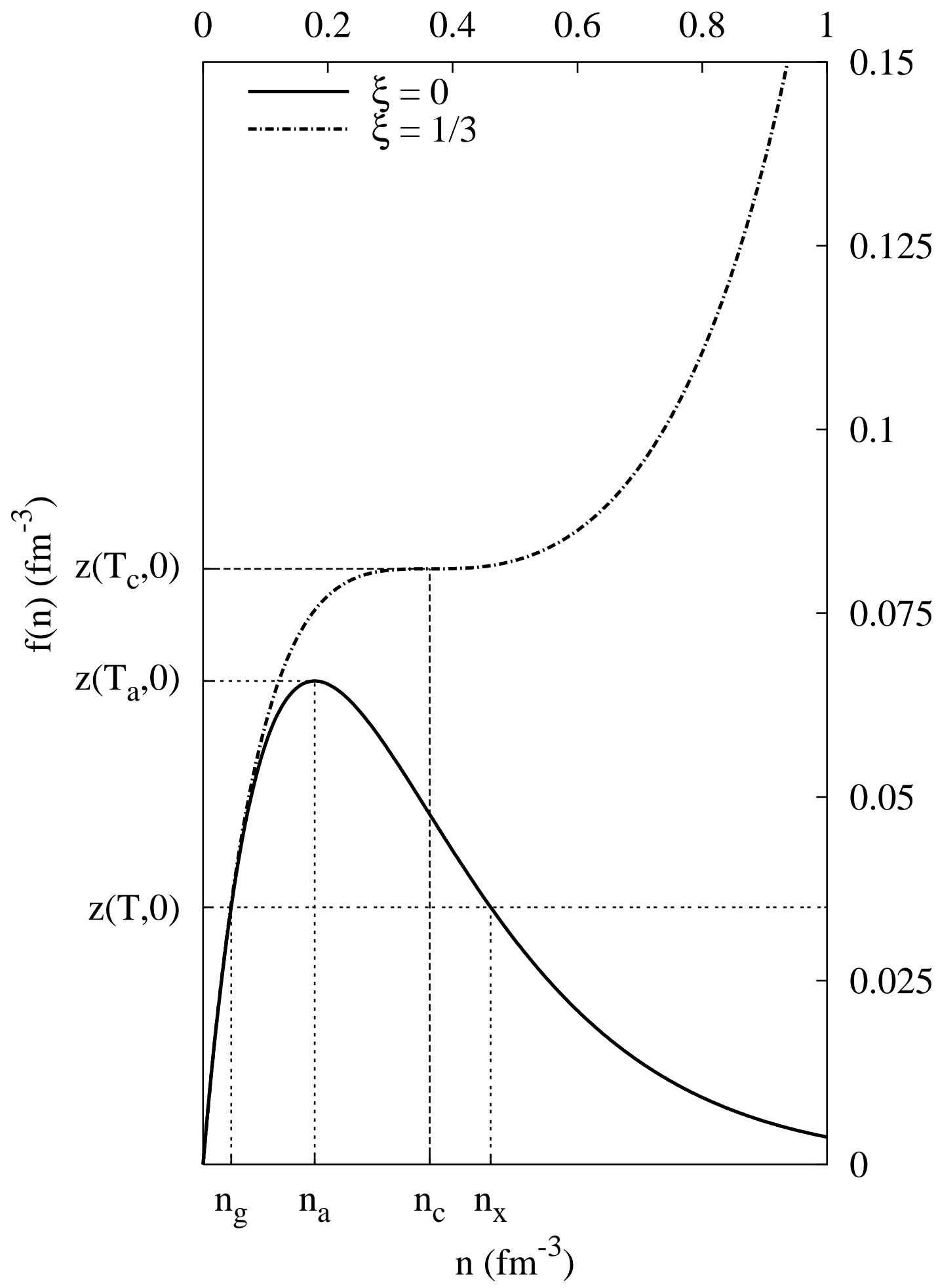

FIG. 2. The dependence of the r.h.s. of the equation (14) $f(n)$ on the variable $n$ at $\mu=0$ for $\xi(T)=0$ and $\xi(T)=1 / 3$ at $T=T_{a}=128 \mathrm{MeV}$ and $T=T_{c}=136 \mathrm{MeV}$, respectively. The behavior of $f(n)$ for $\xi(T)<0(\xi(T)>1 / 3)$ is qualitatively the same as for $\xi(T)=0(\xi(T)=1 / 3)$. 


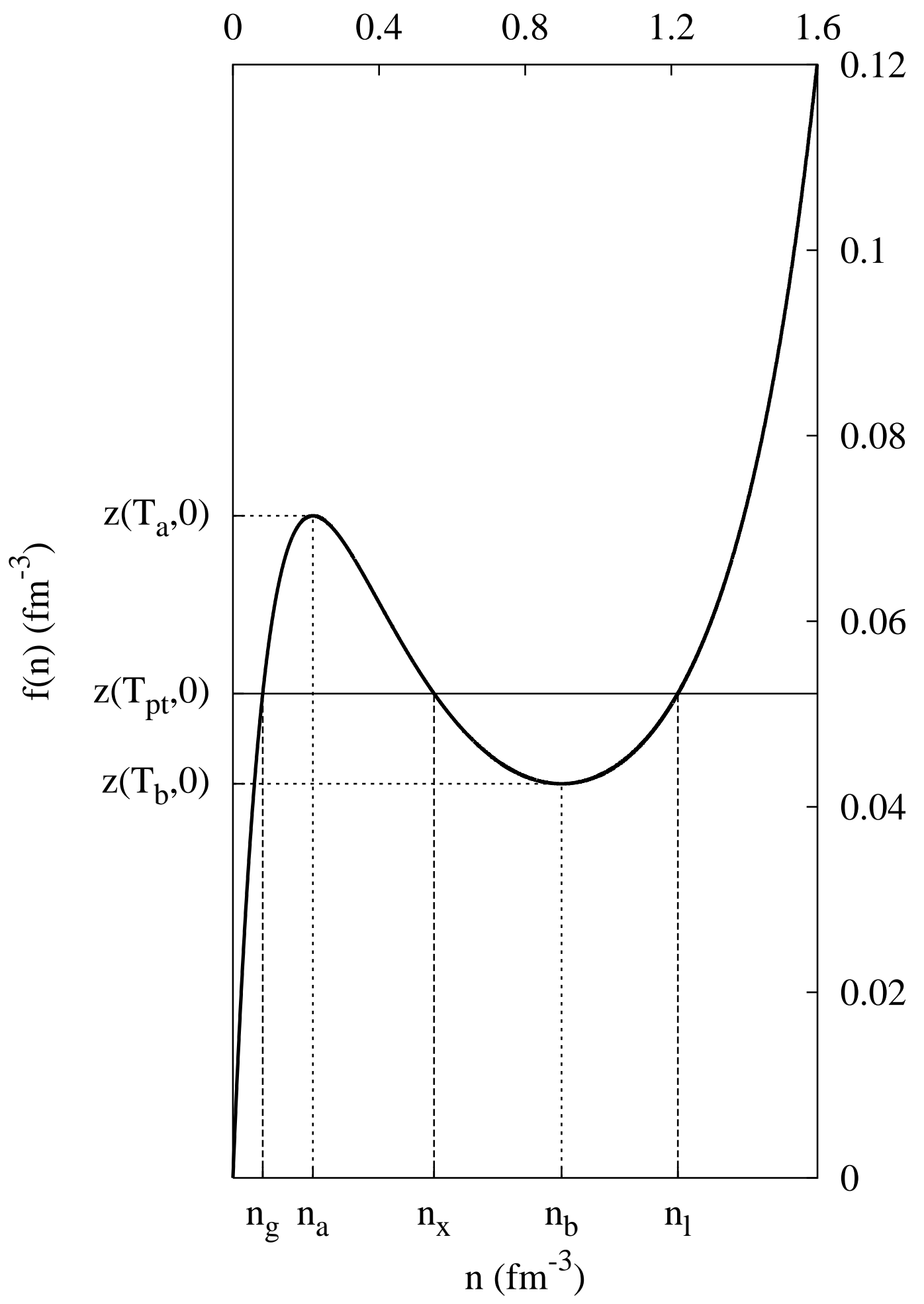

FIG. 3. The dependence of the r.h.s. of the equation (14) $f(n)$ on the variable $n$ at $\mu=0$ for $\xi(T)=0.21$ at the temperature $T=T_{p t}=120 \mathrm{MeV}$. The behavior of $f(n)$ for any $\xi(T)$ from the interval $0<\xi(T)<1 / 3$ at any temperature is qualitatively the same. 


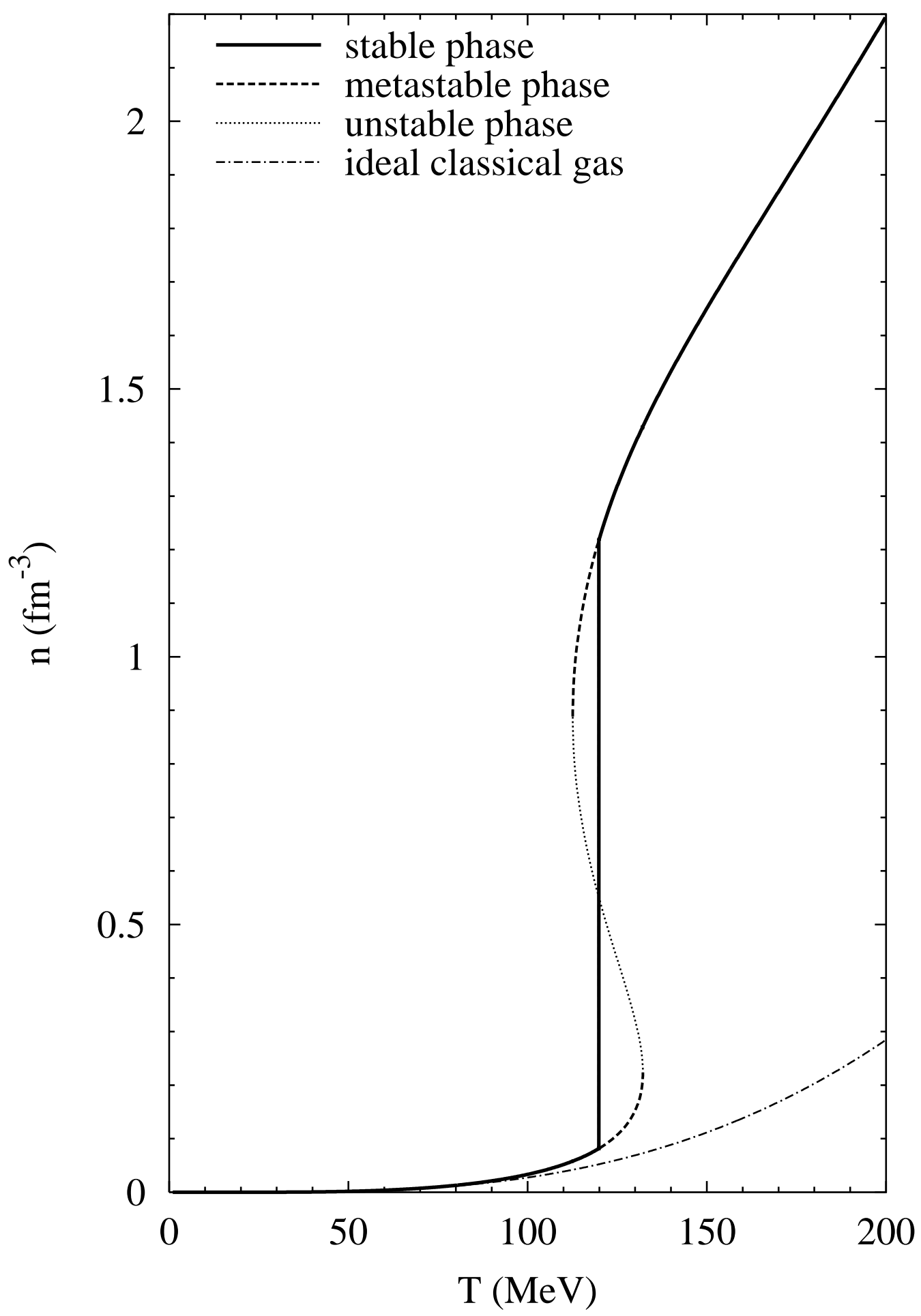

FIG. 4. The dependence of the pion density on the temperature at chemical equilibrium $\mu=0$ for $\xi(T)=0.21$. The density of the ideal classical pion gas is shown for comparison. 